\title{
The environmental impact of inhalers for asthma; a green challenge and a golden opportunity
}

\author{
Alex Wilkinson ${ }^{1}$ and Ashley Woodcock ${ }^{2}$ \\ ${ }^{1}$ East and North Hertfordshire NHS Trust \\ ${ }^{2}$ Manchester University NHS Foundation Trust
}

October 6, 2021

\begin{abstract}
The propellants used in metered-dose inhalers (MDIs) are powerful greenhouse gases, which account for approximately $13 \%$ of the NHS's carbon footprint related to the delivery of care. Most MDI use is in salbutamol relievers in patients with poorly controlled disease. The UK lags behind in this regard with greater reliance on salbutamol MDI and correspondingly greater greenhouse gas emissions; roughly treble our European neighbours'. There has been a broad switch towards MDIs in the UK over the last 20 years to reduce financial costs such that two-thirds of asthma patients in the UK are on treatment dominated by salbutamol MDI. Strategies that replace overuse of reliever MDIs with regimes emphasising inhaled corticosteroids have the potential to improve asthma control alongside significant reductions in greenhouse gas emissions. Real-world evidence shows that once-daily long-acting combination dry-powder inhalers can improve compliance, asthma control and reduce the carbon footprint of care. Similarly, maintenance and reliever therapy (MART) which uses combination reliever and inhaled steroids in one device (usually a dry-powder inhaler) can simplify therapy, improve asthma control and reduce greenhouse gas emissions. Both treatment strategies are popular with patients, most of whom are willing to change treatment to reduce their carbon footprint. By focussing on patients who are currently using high amounts of salbutamol MDI, and prioritising inhaled steroids via dry-powder inhalers, there are golden opportunities to make asthma care more effective, safer and greener.
\end{abstract}

\section{Hosted file}

BJCP review submitted version.docx available at https://authorea.com/users/439660/articles/ 540539-the-environmental-impact-of-inhalers-for-asthma-a-green-challenge-and-a-goldenopportunity 